\title{
HILBERT-SCHMIDT HANKEL OPERATORS ON THE BERGMAN SPACE
}

\author{
KEHE ZHU
}

(Communicated by John B. Conway)

\begin{abstract}
We show that there are no nonzero Hilbert-Schmidt Hankel operators on the Bergman space of the open unit ball in $\mathbf{C}^{n}$ with antiholomorphic symbols when $n \geq 2$.
\end{abstract}

\section{INTRODUCTION}

Let $B_{n}$. be the open unit ball in $C^{n}$ with (normalized) volume measure $d v(z)$. The Bergman space $L_{a}^{2}\left(B_{n}\right)$ of $B_{n}$ is the closed subspace of $L^{2}\left(B_{n}, d v\right)$ consisting of holomorphic functions. The Bergman projection $P$ is the orthogonal projection from $L^{2}\left(B_{n}, d v\right)$ onto $L_{a}^{2}\left(B_{n}\right)$. Given $f \in L^{2}\left(B_{n}, d v\right)$, the Hankel operator (possibly unbounded) $H_{f}: L_{a}^{2}\left(B_{n}\right) \rightarrow L_{a}^{2}\left(B_{n}\right)^{\perp}$ is defined via the Bergman projection $P$ as follows:

$$
H_{f} g=(I-P)(f g), \quad g \in L_{a}^{2}\left(B_{n}\right),
$$

where $I$ is the identity operator on $L^{2}\left(B_{n}, d v\right) . H_{f}$ is densely defined. $f$ is called the symbol of $H_{f}$. It is clear that if $f$ is holomorphic, then $H_{f}=0$. The study of Hankel operators on the Bergman space of the open unit disc $\mathbf{D}$ in the complex plane $\mathbf{C}$ was initiated in [4], and further pursued in [3], [5], [10], [12]. Hankel operators on the Bergman space of bounded symmetric domains were studied in [6], [7]. It was shown in [7] that if $f$ is holomorphic in $B_{n}$, then $H_{\bar{f}}$ is bounded if and only if $f$ is in the Bloch space [11]; $H_{\bar{f}}$ is compact if and only if $f$ is in the little Bloch space. It was shown in [3] that for $1<p<+\infty$ and $f$ holomorphic in $\mathbf{D}, H_{\bar{f}}$ is in the Schatten ideal $S_{p}$ if and only if $f$ is in the Besov space $B_{p}$ consisting of holomorphic functions $g$ on $\mathbf{D}$ such that

$$
\int_{\mathbf{D}}\left(1-|z|^{2}\right)^{p}\left|g^{\prime}(z)\right|^{p} \frac{d A(z)}{\left(1-|z|^{2}\right)^{2}}<+\infty,
$$

Received by the editors May 13, 1989.

1980 Mathematics Subject Classification (1985 Revision). Primary 47B10, 47B35; Secondary 30H05, 47B38.

Research supported in part by the National Science Foundation. 
where $d A$ is the (normalized) area measure on D. In particular, $H_{\bar{f}}$ is HilbertSchmidt if and only if $f$ is in the Dirichlet space consisting of analytic functions $g$ on D with

$$
\int_{\mathbf{D}}\left|g^{\prime}(z)\right|^{2} d A(z)<+\infty .
$$

The main purpose of the paper is to prove the following results.

Theorem. When $n \geq 2$, there are no nonzero Hilbert-Schmidt Hankel operators on $L_{a}^{2}\left(B_{n}\right)$ with antiholomorphic symbols.

The idea of the proof is as follows. First we'll observe that the space $X$ of holomorphic functions $f$ in $B_{n}$ such that $H_{\bar{f}}$ is Hilbert-Schmidt is an invariant Hilbert space. Then we show that every nontrivial invariant Banach space of holomorphic functions in $B_{n}$ must contain all polynomials. Finally we show that for $n \geq 2$, the polynomial $z_{1}$ is not in $X$, thus $X$ consists of only the constant functions. This proof will actually give us a little more.

Theorem. If $n>1$ and $p \leq 2 n$, then there are no nonzero Hankel operators in $S_{p}$ with antiholomorphic symbols in $B_{n}$.

\section{A TRACE ESTIMATE FOR HANKel OPERATORS}

Recall that a linear operator $A$ on a Hilbert space $H$ is Hilbert-Schmidt if

$$
\|A\|_{2}^{2}=\sum_{k=1}^{\infty}\left\|A e_{k}\right\|^{2}=\sum_{k, m=1}^{\infty}\left|\left\langle A e_{k}, e_{m}\right\rangle\right|^{2}<+\infty
$$

for any (or some) orthonormal basis $\left\{e_{k}\right\}$ of $H$. A positive operator $A$ on $H$ is in the trace class if

$$
\operatorname{tr}(A)=\sum_{k=1}^{\infty}\left\langle A e_{k}, e_{k}\right\rangle<+\infty
$$

for any (or some) orthonormal basis $\left\{e_{k}\right\}$ of $H \cdot \operatorname{tr}(A)$ is independent of the choice of the orthonormal basis $\left\{e_{k}\right\}$. Clearly, $A$ is Hilbert-Schmidt if and only if $A^{*} A$ is in the trace class. Moreover, $\|A\|_{2}^{2}=\operatorname{tr}\left(A^{*} A\right)$. In general, we say that a bounded linear operator $A$ is in the Schatten ideal $S_{p}$ if $\left(A^{*} A\right)^{p / 2}$ is in the trace class. We let $\|A\|_{p}=\left[\operatorname{tr}\left(A^{*} A\right)^{p / 2}\right]^{1 / p}$. For more information on Schatten ideals, see [8].

We say that a Hankel operator $H_{f}: L_{a}^{2}\left(B_{n}\right) \rightarrow L_{a}^{2}\left(B_{n}\right)^{\perp}$ is Hilbert-Schmidt if $H_{f}^{*} H_{f}: L_{a}^{2}\left(B_{n}\right) \rightarrow L_{a}^{2}\left(B_{n}\right)$ is in the trace class. We say that $H_{f}$ is in $S_{p}$ if $\operatorname{tr}\left(H_{f}^{*} H_{f}\right)^{p / 2}<+\infty$.

Let

$$
K(z, w)=\frac{1}{(1-\langle z, w\rangle)^{n+1}}
$$

be the Bergman kernel of $B_{n}$. The Bergman projection $P$ is given by

$$
P f(z)=\int_{B_{n}} K(z, w) f(w) d v(w), \quad f \in L^{2}\left(B_{n}, d v\right) .
$$


For $\lambda \in B_{n}$, let

$$
k_{\lambda}(z)=\frac{K(z, \lambda)}{\sqrt{K(\lambda, \lambda)}}, \quad z \in B_{n} .
$$

$k_{\lambda}$ is a unit vector in $L_{a}^{2}\left(B_{n}\right)$. For any $f$ in $L^{1}\left(B_{n}, d v\right)$, we define a function $\tilde{f}$ on $B_{n}$ as follows:

$$
\tilde{f}(z)=\left\langle f k_{z}, k_{z}\right\rangle=\int_{B_{n}} f(w)\left|k_{z}(w)\right|^{2} d v(w) .
$$

$\tilde{f}$ is called the Berezin transform of $f[6,7,12]$. By a change of variable, we have

$$
\tilde{f}(z)=\int_{B_{n}} f\left(\varphi_{z}(w)\right) d v(w),
$$

where $\varphi_{z}$ is the biholomorphic mapping from $B_{n}$ onto $B_{n}$ defined in 2.2 of [9]. The reproducing property of $K(z, w)$ easily implies that $\tilde{f}=f$ for $f$ in $L_{a}^{2}\left(B_{n}\right)$.

Theorem 1. There exists a constant $C>0$ such that

$$
\begin{aligned}
& \int_{B_{n}}\left(\widetilde{\left.f\right|^{2}}(z)-|\tilde{f}(z)|^{2}\right) K(z, z) d v(z) \leq C \operatorname{tr}\left(H_{f}^{*} H_{f}+H_{f}^{*} H_{\bar{f}}\right), \\
& \operatorname{tr}\left(H_{f}^{*} H_{f}+H_{\frac{f}{f}}^{*} H_{\bar{f}}\right) \leq C \int_{B_{n}}\left(\widetilde{|f|^{2}}(z)-|\tilde{f}(z)|^{2}\right) K(z, z) d v(z)
\end{aligned}
$$

for all $f$ in $L^{2}\left(B_{n}, d v\right)$. Moreover, if $f \in L_{a}^{2}\left(B_{n}\right)$, then

$$
\operatorname{tr}\left(H_{f}^{*} H_{-}\right)=\int_{B_{n}}\left(\widetilde{|f|^{2}}(z)-|\tilde{f}(z)|^{2}\right) K(z, z) d v(z) .
$$

Proof. By Lemma 13 of [13],

$$
\begin{aligned}
\operatorname{tr}\left(H_{f}^{*} H_{f}\right) & =\int_{B_{n}}\left\langle H_{f}^{*} H_{f} K(\cdot, z), K(\cdot, z)\right\rangle d v(z) \\
& =\int_{B_{n}}\left\|H_{f} k_{z}\right\|^{2} K(z, z) d v(z) \\
& =\int_{B_{n}}\left\|(I-P)\left(f k_{z}\right)\right\|^{2} K(z, z) d v(z) \\
& =\int_{B_{n}}\left(\left\|f k_{z}\right\|^{2}-\left\|P\left(f k_{z}\right)\right\|^{2}\right) K(z, z) d v(z) \\
& =\int_{B_{n}}\left(|f|^{2}(z)-\left\|P\left(f k_{z}\right)\right\|^{2}\right) K(z, z) d v(z) .
\end{aligned}
$$

By the Cauchy-Schwarz inequality, we have

$$
|\tilde{f}(z)|=\left|\left\langle f k_{z}, k_{z}\right\rangle\right|=\left|\left\langle P\left(f k_{z}\right), k_{z}\right\rangle\right| \leq\left\|P\left(f k_{z}\right)\right\| .
$$

Therefore,

$$
\operatorname{tr}\left(H_{f}^{*} H_{f}\right) \leq \int_{B_{n}}\left(\widetilde{|f|^{2}}(z)-|\tilde{f}(z)|^{2}\right) K(z, z) d v(z)
$$


Replace $f$ by $\bar{f}$; then

$$
\operatorname{tr}\left(H_{f}^{*} H_{\bar{f}}\right) \leq \int_{B_{n}}\left(\widetilde{|f|^{2}}(z)-|\tilde{f}(z)|^{2}\right) K(z, z) d v(z)
$$

since $\tilde{\bar{g}}=\overline{\tilde{g}}$ for any $g$ in $L^{1}\left(B_{n}, d v\right)$. Thus

$$
\operatorname{tr}\left(H_{f}^{*} H_{f}+H_{\bar{f}}^{*} H_{\bar{f}}\right) \leq 2 \int_{B_{n}}\left(\widetilde{|f|^{2}}(z)-|\tilde{f}(z)|^{2}\right) K(z, z) d v(z) .
$$

On the other hand, by Theorem 3 of [7],

$$
\widetilde{|f|^{2}}(z)-|\tilde{f}(z)|^{2} \leq 2(1+\sqrt{2})^{2}\left(\left\|H_{f} k_{z}\right\|^{2}+\left\|H_{\bar{f}} k_{z}\right\|^{2}\right),
$$

thus

$$
\begin{aligned}
& \int_{B_{n}}\left(\widetilde{|f|^{2}}(z)-|\tilde{f}(z)|^{2}\right) K(z, z) d v(z) \\
& \quad \leq 2(1+\sqrt{2})^{2} \int_{B_{n}}\left(\left\|H_{f} k_{z}\right\|^{2}+\left\|H_{\bar{f}} k_{z}\right\|^{2}\right) K(z, z) d v(z) \\
& \quad=2(1+\sqrt{2})^{2} \operatorname{tr}\left(H_{f}^{*} H_{f}+H_{\bar{f}}^{*} H_{\bar{f}}\right)
\end{aligned}
$$

and the first part of Theorem 1 is proved.

If $f$ is holomorphic, then $H_{f}=0$ and $P\left(\bar{f} k_{z}\right)(w)=\bar{f}(z) k_{z}(w)$, so

$$
\left\|P\left(\bar{f} k_{z}\right)\right\|=\left\|\bar{f}(z) k_{z}\right\|=|\bar{f}(z)|=|f(z)|=|\tilde{f}(z)| .
$$

It follows that

$$
\begin{aligned}
\operatorname{tr}\left(H_{\bar{f}}^{*} H_{\bar{f}}\right) & =\int_{B_{n}}\left(\widetilde{|f|^{2}}(z)-\left\|P\left(\bar{f} k_{z}\right)\right\|^{2}\right) K(z, z) d v(z) \\
& =\int_{B_{n}}\left(\widetilde{|f|^{2}}(z)-|\tilde{f}(z)|^{2}\right) K(z, z) d v(z),
\end{aligned}
$$

completing the proof of Theorem 1 .

Corollary 2. Given $f$ in $L^{2}\left(B_{n}, d v\right)$, then $H_{f}$ and $H_{\bar{f}}$ are both Hilbert-Schmidt if and only if

$$
\int_{B_{n}}\left(\widetilde{|f|^{2}}(z)-|\tilde{f}(z)|^{2}\right) K(z, z) d v(z)<+\infty
$$

\section{INVARIANT BANACH SPACES OF HOLOMORPHIC FUNCTIONS}

The following technical lemma will be needed in the proof of the main results. We isolate its proof in this section.

Lemma 3. Suppose $X$ is a linear space of holomorphic functions in $B_{n}$ with a complete seminorm \|\| . Assume that $X$ satisfies the following conditions:

(1) $X$ contains a nonconstant function;

(2) $f \circ \varphi \in X$ and $\|f \circ \varphi\|=\|f\|$ whenever $f \in X$ and $\varphi \in \operatorname{Aut}\left(B_{n}\right)$, where $\operatorname{Aut}\left(B_{n}\right)$, is the group of biholomorphic mappings of $B_{n}$;

(3) $\left(\theta_{1}, \ldots, \theta_{n}\right) \mapsto f\left(z_{1} e^{i \theta_{1}}, \ldots, z_{n} e^{i \theta_{n}}\right):[0,2 \pi]^{n} \rightarrow X$ is continuous for each $f$ in $X$.

Then $X$ contains all polynomials. 
Proof. Let $f$ be a nonconstant function in $X$. Write

$$
f(z)=\sum_{\alpha} a_{\alpha} z^{\alpha}
$$

where $\alpha=\left(\alpha_{1}, \ldots, \alpha_{n}\right)$ is an $n$-tuple of nonnegative integers and $z^{\alpha}=$ $z_{1}^{\alpha_{1}} \cdots z_{n}^{\alpha_{n}}$. Since $f$ is nonconstant, there exists $\alpha_{0} \neq 0$ such that $a_{\alpha_{0}} \neq 0$. Write $\alpha_{0}=\left(k_{1}, \ldots, k_{n}\right)$, then it is easy to see that

$$
a_{\alpha_{0}} z^{\alpha_{0}}=\frac{1}{(2 \pi)^{n}} \int_{0}^{2 \pi} \cdots \int_{0}^{2 \pi} f\left(z_{1} e^{i \theta_{1}}, \ldots, z_{n} e^{i \theta_{n}}\right) e^{-i k_{1} \theta_{1}} \cdots e^{-i k_{n} \theta_{n}} d \theta_{1} \cdots d \theta_{n} .
$$

By conditions 3 and 2, $a_{\alpha_{0}} z^{\alpha_{0}} \in X$. Since $a_{\alpha_{0}} \neq 0$, we have $z^{\alpha_{0}} \in X$.

Let $k=k_{1}+\cdots+k_{n}>0$. We show that $z_{1}^{k} \in X$. Let $U=\left(u_{i j}\right)_{n \times n}$ be a unitary matrix each of whose entries in the first column is $\frac{1}{\sqrt{n}}$, then

$$
z^{\alpha_{0}} \circ U(z)=\left(u_{11} z_{1}+\cdots+u_{1 n} z_{n}\right)^{k_{1}} \cdots\left(u_{n 1} z_{1}+\cdots+u_{n n} z_{n}\right)^{k_{n}}=\left(\frac{1}{\sqrt{n}}\right)^{k} z_{1}^{k}+R(z) \text {, }
$$

where $R(z)$ is a homogeneous polynomial of degree $k$ each of whose nonzero terms contains at least one positive power of $z_{2}, \ldots, z_{n}$. It follows that

$$
\int_{0}^{2 \pi} \cdots \int_{0}^{2 \pi} R\left(z_{1}, z_{2} e^{i \theta_{2}}, \ldots, z_{n} e^{i \theta_{n}}\right) d \theta_{2} \cdots d \theta_{n}=0 .
$$

Therefore,

$$
\frac{1}{(2 \pi)^{n-1}} \int_{0}^{2 \pi} \cdots \int_{0}^{2 \pi} z^{\alpha_{0}} \circ U\left(z_{1}, z_{2} e^{i \theta_{2}}, \ldots, z_{n} e^{i \theta_{n}}\right) d \theta_{2} \cdots d \theta_{n}=\left(\frac{1}{\sqrt{n}}\right)^{k} z_{1}^{k}
$$

is in $X$.

Recall that for each $\lambda \in B_{n}, \varphi_{\lambda}$ is the biholomorphic mapping on $B_{n}$ defined in 2.2 of [9]. If $\lambda=(r, 0, \ldots, 0)$ for $r \in(-1,1)$, we write $\varphi_{\lambda}=\varphi_{r}$. It is easy to see (from the definition of $\varphi_{\lambda}$ ) that

$$
\varphi_{r}\left(z_{1}, \ldots, z_{n}\right)=\left(\frac{r-z_{1}}{1-r z_{1}}, \frac{-\sqrt{1-r^{2}} z_{2}}{1-r z_{1}}, \ldots, \frac{-\sqrt{1-r^{2}} z_{n}}{1-r z_{1}}\right) .
$$

By $1, z_{1}^{k} \circ \varphi_{r} \in X$ for all $r \in(-1,1)$, thus

$$
\left(\frac{r-z_{1}}{1-r z_{1}}\right)^{k}=r^{k}-k r^{k-1}\left(1-r^{2}\right) z_{1}+\cdots
$$

is in $X$. But

$$
\frac{1}{2 \pi} \int_{0}^{2 \pi}\left(\frac{r-z_{1} e^{i \theta_{1}}}{1-r z_{1} e^{i \theta_{1}}}\right)^{k} e^{-i \theta_{1}} d \theta_{1}=-k r^{k-1}\left(1-r^{2}\right) z_{1},
$$

so $z_{1} \in X$.

Since

$$
z_{1} \circ \varphi_{r}(z)=\frac{r-z_{1}}{1-r z_{1}}=r-\sum_{j=1}^{\infty} r^{j-1}\left(1-r^{2}\right) z_{1}^{j}
$$


the integral

$$
\frac{1}{2 \pi} \int_{0}^{2 \pi} z_{1} \circ \varphi_{r}\left(z_{1} e^{i \theta_{1}}, z_{2}, \ldots, z_{n}\right) e^{-i m \theta_{1}} d \theta_{1}
$$

gives $z_{1}^{m} \in X$ for all $m \geq 0$.

Finally, let $V=\left(v_{i j}\right)_{n \times n}$ be a unitary matrix each of whose entries in the first row is $\frac{1}{\sqrt{n}}$, then

$$
\begin{aligned}
z_{1}^{m} \circ V(z) & =\left(\frac{1}{\sqrt{n}} z_{1}+\cdots+\frac{1}{\sqrt{n}} z_{n}\right)^{m} \\
& =\left(\frac{1}{\sqrt{n}}\right)^{m} \sum_{|\alpha|=m} \frac{m !}{\alpha !} z^{\alpha},
\end{aligned}
$$

where $\alpha !=\alpha_{1} ! \cdots \alpha_{n} !$. For any $|\alpha|=m$, the integral

$$
\frac{1}{(2 \pi)^{n}} \int_{0}^{2 \pi} \cdots \int_{0}^{2 \pi} z_{1}^{m} \circ V\left(z_{1} e^{i \theta_{1}}, \ldots, z_{n} e^{i \theta_{n}}\right) e^{-i \alpha_{1} \theta_{1}} \cdots e^{-i \alpha_{n} \theta_{n}} d \theta_{1} \cdots d \theta_{n}
$$

gives $z^{\alpha} \in X$. Since $m$ is arbitrary, we have completed the proof of Lemma 3.

Remark. The proof of Lemma 3 generalizes the proof of Proposition 2 in [2]. The general theory of invariant Banach spaces of holomorphic functions in $\mathbf{D}$ was developed in [2], [1].

\section{Proof of the MAIN Results}

We now prove

Theorem 4. If $n \geq 2$, then there are no nonzero Hilbert-Schmidt Hankel operators on $L_{a}^{2}\left(B_{n}\right)$ with antiholomorphic symbols in $B_{n}$.

Proof. For any $f$ in $L_{a}^{2}\left(B_{n}\right)$, we have

$$
f(z)-f(0)=\int_{B_{n}} \frac{f(w)-f(0)}{(1-\langle z, w\rangle)^{n+1}} d v(w) .
$$

Differentiating under the integral sign, we have

$$
\frac{\partial f}{\partial z_{i}}(0)=(n+1) \int_{B_{n}} \bar{w}_{i}(f(w)-f(0)) d v(w), \quad 1 \leq i \leq n .
$$

It follows that

$$
\begin{aligned}
\sum_{i=1}^{n}\left|\frac{\partial f}{\partial z_{i}}(0)\right|^{2} & \leq(n+1)^{2} \int_{B_{n}}|w|^{2}|f(w)-f(0)|^{2} d v(w) \\
& \leq(n+1)^{2} \int_{B_{n}}|f(w)-f(0)|^{2} d v(w) .
\end{aligned}
$$

It is easy to see that

$$
\int_{B_{n}}|f(w)-f(0)|^{2} d v(w)=\widetilde{|f|^{2}}(0)-|\tilde{f}(0)|^{2}
$$


and

$$
\Delta\left(|f|^{2}\right)(0)=4 \sum_{i=1}^{n}\left|\frac{\partial f}{\partial z_{i}}(0)\right|^{2},
$$

where $\Delta$ is the (usual) Laplacian on $B_{n}$. Therefore,

$$
\Delta\left(|f|^{2}\right)(0) \leq 4(n+1)^{2}\left(\widetilde{|f|^{2}}(0)-|\tilde{f}(0)|^{2}\right) .
$$

Replacing $f$ by $f \circ \varphi_{z}$, then

$$
\left.\tilde{\Delta}\left(|f|^{2}\right)(z) \leq 4(n+1)^{2} \widetilde{\left(|f|^{2}\right.}(z)-|\tilde{f}(z)|^{2}\right)
$$

for all $f \in L_{a}^{2}\left(B_{n}\right)$, where $\tilde{\Delta}$ is the invariant Laplacian of $B_{n}$ [9]. By Theorem 1 , we have

$$
\int_{B_{n}} \tilde{\Delta}\left(|f|^{2}\right)(z) K(z, z) d v(z) \leq 4(n+1)^{2} \operatorname{tr}\left(H_{\bar{f}}^{*} H_{\bar{f}}\right) .
$$

Now let $X$ be the space of holomorphic functions $f$ on $B_{n}$ with

$$
\|f\|_{X}=\left[\int_{B_{n}} \tilde{\Delta}\left(|f|^{2}\right)(z) K(z, z) d v(z)\right]^{1 / 2}<+\infty,
$$

then \|\|$_{X}$ is an invariant complete seminorm on $X$, and $X$ contains $f$ if $H_{\bar{f}}$ is Hilbert-Schmidt. Thus Theorem 4 will be proved (by Lemma 3 ) if we can show that condition 3 of Lemma 3 is satisfied and $z_{1} \bar{\epsilon} X$.

We first check that $z_{1} \bar{\in} X$ if $n \geq 2$. By [9],

$$
\begin{aligned}
\int_{B_{n}} \tilde{\Delta} & \left(\left|z_{1}\right|^{2}\right)(z) K(z, z) d v(z) \\
& =4 \int_{B_{n}} \frac{\left(1-|z|^{2}\right)\left(1-\left|z_{1}\right|^{2}\right)}{\left(1-|z|^{2}\right)^{n+1}} d v(z) \\
& =4 \int_{B_{n}} \frac{1-\left|z_{1}\right|^{2}}{\left(1-|z|^{2}\right) n} d v(z) \\
& \geq 4 \int_{B_{n}} \frac{d v(z)}{\left(1-|z|^{2}\right)^{n-1}}=+\infty
\end{aligned}
$$

if $n \geq 2$.

It remains to check that $X$ satisfies condition 3 of Lemma 3. For any $\theta=$ $\left(\theta_{1}, \ldots, \theta_{n}\right) \in[0,2 \pi]^{n}$, let $U_{\theta}$ be the operator defined by

$$
U_{\theta} f(z)=f\left(z_{1} e^{i \theta_{1}}, \ldots, z_{n} e^{i \theta_{n}}\right)
$$

then we have to show that

$$
\left\|U_{\theta} f-f\right\|_{X}=\left[\int_{B_{n}} \tilde{\Delta}\left(\left|U_{\theta} f-f\right|^{2}\right)(z) K(z, z) d v(z)\right]^{1 / 2} \rightarrow 0 \quad(\theta \rightarrow 0)
$$


for any fixed $f$ in $X$. Write $B_{n}=\{z:|z|<r\} \cup\{z: r \leq|z|<1\}$ and apply the triangle inequality, then

$$
\begin{aligned}
\left\|U_{\theta} f-f\right\|_{X} \leq & {\left[\int_{|z|<r} \tilde{\Delta}\left(\left|U_{\theta} f-f\right|^{2}\right)(z) K(z, z) d v(z)\right]^{1 / 2} } \\
& +\left[\int_{r \leq|z|<1} \tilde{\Delta}\left(\left|U_{\theta} f-f\right|^{2}\right)(z) K(z, z) d v(z)\right]^{1 / 2} .
\end{aligned}
$$

The first term above tends to 0 (for any $r \in(0,1)$ ) as $\theta \rightarrow 0$ (by uniform convergence). The second term above can be made arbitrarily small by choosing $r$ close enough to 1 because

$$
\int_{B_{n}} \tilde{\Delta}\left(|f|^{2}\right)(z) K(z, z) d v(z)<+\infty,
$$

and

$$
\left[\tilde{\Delta}\left(\left|U_{\theta} f-f\right|^{2}\right)(z)\right]^{1 / 2} \leq\left[\tilde{\Delta}\left(\left|U_{\theta} f\right|^{2}\right)(z)\right]^{1 / 2}+\left[\tilde{\Delta}\left(|f|^{2}\right)(z)\right]^{1 / 2},
$$

and the measure $K(z, z) d v(z)$ is rotation invariant. This completes the proof of Theorem 4.

For any $1 \leq p<+\infty$, Lemma 13 of [13] gives

$$
\left\|H_{f}\right\|_{p}^{p}=\operatorname{tr}\left(\left(H_{f}^{*} H_{f}\right)^{p / 2}\right)=\int_{B_{n}}\left\langle\left(H_{f}^{*} H_{f}\right)^{p / 2} k_{z}, k_{z}\right\rangle K(z, z) d v(z) .
$$

If $p \geq 2$, then by Proposition 6.4 of [3],

$$
\left\|H_{f}\right\|_{p}^{p} \geq \int_{B_{n}}\left\langle H_{f}^{*} H_{f} k_{z}, k_{z}\right\rangle^{p / 2} K(z, z) d v(z) .
$$

If $f$ is further antiholomorphic, then

$$
\left\langle H_{\bar{f}}^{*} H_{\bar{f}} k_{z}, k_{z}\right\rangle=\left\|H_{\bar{f}} k_{z}\right\|^{2}=\widetilde{|f|^{2}}(z)-|\tilde{f}(z)|^{2},
$$

and

$$
\begin{aligned}
\left\|H_{\bar{f}}\right\|_{p}^{p} & \geq \int_{B_{n}}\left(\widetilde{\left.f f\right|^{2}}(z)-|\tilde{f}(z)|^{2}\right)^{p / 2} K(z, z) d v(z) \\
& \geq\left[\frac{1}{2(n+1)}\right]^{p} \int_{B_{n}}\left(\tilde{\Delta}\left(|f|^{2}\right)(z)\right)^{p / 2} K(z, z) d v(z) .
\end{aligned}
$$

Let $X_{p}$ be the space of holomorphic functions $f$ on $B_{n}$ such that

$$
\|f\|_{p}^{p}=\int_{B_{n}}\left[\tilde{\Delta}\left(|f|^{2}\right)(z)\right]^{p / 2} K(z, z) d v(z)<+\infty .
$$


Then $X_{p}$ contains all functions $f$ such that $H_{\bar{f}} \in S_{p}$, and $X_{p}$ satisfies conditions 2 and 3 of Lemma 3 (see the proof of Theorem 4). Since

$$
\begin{aligned}
\int_{B_{n}}[ & \left.\tilde{\Delta}\left(\left|z_{1}\right|^{2}\right)(z)\right]^{p / 2} K(z, z) d v(z) \\
& =\int_{B_{n}}\left[4\left(1-|z|^{2}\right)\left(1-\left|z_{1}\right|^{2}\right)\right]^{p / 2} K(z, z) d v(z) \\
& =2^{p} \int_{B_{n}} \frac{\left(1-\left|z_{1}\right|^{2}\right)^{p / 2}}{\left(1-|z|^{2}\right)^{n+1-p / 2}} d v(z)=+\infty
\end{aligned}
$$

if $n>1$ and $2 \leq p \leq 2 n$. It follows from Lemma 3 that $X_{p}$ contains only the constant functions when $n \geq 2$ and $2 \leq p \leq 2 n$. Since $S_{p} \subset S_{q}$ for $0<p<q<+\infty$, we have proved the following.

Theorem 5. If $n>1$ and $0<p \leq 2 n$, then there are no nonzero Hankel operators in $S_{p}$ with antiholomorphic symbols in $B_{n}$.

\section{REMARKS}

The analysis in the previous sections easily implies the following: If $2 \leq p<$ $+\infty$ and $H_{f}, H_{\bar{f}}$ are in $S_{p}$, then $\left[|\widetilde{f}|^{2}(z)-|\tilde{f}(z)|^{2}\right]^{1 / 2}$ is in

$$
L^{p}\left(B_{n}, K(z, z) d v(z)\right) .
$$

Moreover, the converse is true for $p=2$. This leads to the following.

Conjecture 1. If $1 \leq p<+\infty$, then $H_{f}$ and $H_{\bar{f}}$ are in $S_{p}$ if and only if $\left[|\widetilde{f}|^{2}(z)-|\tilde{f}(z)|^{2}\right]^{1 / 2}$ is in $L^{p}\left(B_{n}, K(z, z) d v(z)\right)$.

The corresponding results for Toeplitz operators on the Bergman spaces of bounded symmetric domains were established in [13].

Let $Z_{p}$ be the space of holomorphic functions $f$ in $B_{n}$ such that

$$
\|f\|_{p}^{p}=\int_{B_{n}}\left[\widetilde{\left.f f\right|^{2}}(z)-|\tilde{f}(z)|^{2}\right]^{p / 2} K(z, z) d v(z)<+\infty .
$$

We know that $Z_{p}$ consists of only the constant functions if $n>1$ and $1 \leq p \leq$ $2 n$. On the other hand, when $p$ is large enough, it is easy to see that $z_{1} \in Z_{p}$. Since $Z_{p}$ is an invariant Banach space, Lemma 3 implies that $Z_{p}$ contains all polynomials if $p$ is large. This suggests

Conjecture 2. $Z_{p}$ is nontrivial iff $p>2 n$. When $Z_{p}$ is nontrivial, we have $f \in Z_{p}$ iff $H_{\bar{f}}$ is in $S_{p}$ iff

$$
\int_{B_{n}}\left[\tilde{\Delta}\left(|f|^{2}\right)(z)\right]^{p / 2} K(z, z) d v(z)<+\infty .
$$

We have seen that the theory of Schatten ideal Hankel operators with antiholomorphic symbols is closely related to the theory of invariant Banach spaces 
of holomorphic functions. This theory was discussed in [2] and [1] for the open unit disk D. In higher dimensions, the author has recently obtained the following result [14]: There exists a unique Hilbert space of holomorphic functions $f$ in $B_{n}$ whose (semi-)inner product is invariant under the $\operatorname{group} \operatorname{Aut}\left(B_{n}\right)$ of biholomorphic mappings. When $n=1$, this space is the Dirichlet space consisting of exactly the analytic functions $f$ in $\mathbf{D}$ such that $H_{\bar{f}}$ is HilbertSchmidt. Here exists an interesting difference between dimension 1 and higher dimensions, because when $n \geq 2, H_{\bar{f}}$ is never Hilbert-Schmidt unless $f$ is constant.

\section{ACKNOWLEDGMENT}

The author wishes to thank the referee for several useful suggestions and pointing out an error in the original manuscript.

\section{REFERENCES}

1. J. Arazy and S. Fisher, The uniqueness of the Dirichlet space among Möbius invariant Hilbert spaces, Ill. J. Math. 29 (1985), 449-462.

2. J. Arazy, S. Fisher, and J. Peetre, Möbius invariant function spaces, J. Reine Angew. Math. 363 (1985), 110-145.

3. __ Hankel operators on weighted Bergman spaces, Amer. J. Math. 110 (1988), 989-1054.

4. S. Axler, The Bergman space, the Bloch space, and commutators of multiplication operators, Duke Math. J. 53 (1986), 315-332.

5. S. Axler, Bergman spaces and their operators, Pitman Research Notes in Math. 171 (1988), $1-55$.

6. C. A. Berger, L. A. Coburn, and K. H. Zhu, Function theory in Cartan domains and the Berezin-Toeplitz symbol calculus, Amer. J. Math. 110 (1988), 921-953.

7. D. Békollé, C. A. Berger, L. A. Coburn, and K. H. Zhu, BMO in the Bergmann metric on bounded symmetric domains, J. Funct. Anal. (to appear).

8. I. C. Gohberg and M. G. Krein, Introduction to the theory of linear nonselfadjoint operators, AMS Translations of Mathematical Monographs, 18, 1969.

9. W. Rudin, Function theory in the unit ball of $\mathbf{C}^{n}$, Springer, 1980.

10. K. Stroethoff, Compact Hankel operators on the Bergman space, Ill. J. Math. (to appear).

11. R. Timoney, Bloch functions in several complex variables, I, Bull. London Math. Soc. 12 (1980), 241-267.

12. K. H. Zhu, VMO, ESV, and Toeplitz operators on the Bergman space, Trans. Amer. Math. Soc. 302 (1987), 617-646.

13. _ Positive Toeplitz operators on weighted Bergman spaces of bounded symmetric domains, J. Operator Theory 20 (1988), 329-357.

14. Invariant Hilbert spaces of holomorphic functions in the open unit ball of $\mathbf{C}^{n}$, Trans. Amer. Math. Soc. (to appear).

Department of Mathematics, State University of New York, Albany, New York 12222 